Pacific Journal of Mathematics

SCHRÖDINGER AND DIRAC OPERATORS WITH SINGULAR

Paul Robert Cherno 


\title{
SCHRÖDINGER AND DIRAC OPERATORS WITH SINGULAR POTENTIALS AND HYPERBOLIC EQUATIONS
}

\author{
PaUl R. ChernofF
}

\begin{abstract}
In an earlier paper we employed techniques from the theory of hyperbolic partial differential equations to deduce the self-adjointness of Dirac and Schrödinger operators with smooth potentials. The present paper applies these techniques to operators with singular potentials.
\end{abstract}

1. Introduction. There is a physical idea in back of our arguments which is especially clear in the case of operators of Dirac type. The Dirac operator $H=D+V$, a first-order partial differential operator, is the quantum Hamiltonian governing the dynamics of a relativistic particle in an external electromagnetic field. Intuitively, we expect that $H$ (with domain $C_{0}^{\infty}$ ) should be essentially self-adjoint if the time evolution is determined by the formal differential expression alone-that is, no boundary conditions are needed to tell the particle how to be scattered when it hits a singularity. First of all, we require that the underlying "physical space" be a complete Riemannian manifold so that no finite points are missing. Moreover, in a relativistic system waves propagate at the speed of light; hence compactly supported data are not propagated to infinity in a finite time, and thus no boundary conditions at infinity are required. Finally, suppose that the potential term $V$ is locally well-behaved in a sense which we will make precise later-roughly that everywhere $V$ is locally equal to a potential that requires no special boundary conditions. Then we expect that $H$ is essentially self-adjoint. That is, by exploiting the finite propagation speed of the Dirac equation, we can patch together local good behavior to deduce global good behavior. This is the main result of $\S 2$.

In $\S \S 3$ and 4 we apply analogous ideas to second-order Schrödinger operators, by considering the associated wave equations. Although the underlying ideas are similar to the Dirac case, there are a number of technical complications, some of which are dealt with in the preliminary material in $\S 3$. The conclusion is, roughly, that a Schrödinger operator with a locally well-behaved potential, which does not decrease too rapidly at infinity, is essentially self-adjoint; this global condition on the potential is needed because the nonrelativistic Schrödinger equation has infinite velocity of propagation.

(There is a large literature devoted to conditions which imply the essential self-adjointness of formally symmetric partial differential 
operators. Some recent general references are the books by Schechter [11], Faris [4], and Reed and Simon [10, vol. II].)

2. Dirac operators. We work in the setting of our paper [2]. Let $M$ be a complete Riemannian manifold and $\xi$ a Hermitian vector bundle over $M$. Form the Hilbert space $\boldsymbol{H}=L^{2}(\xi)$ of square integrable sections of $\xi$. Denote by $C_{0}^{\infty}(\xi)$ the $C^{\infty}$ sections of $\xi$ with compact support. We want to discuss perturbations of a Dirac-type operator $D: C_{0}^{\infty}(\xi) \rightarrow C_{0}^{\infty}(\xi)$, a first-order differential operator which we assume is symmetric. For $x \in M$ let $c(x)$ be the local propagation speed associated with $D$ (for the precise definition see [2]; roughly, $c(x)$ is the largest eigenvalue of the symbol of $D$ ). Fix a point $0 \in M$ and let $B_{r}$ be the ball in $M$ with center 0 and radius $r$. Define

$$
c(r)=\sup \left\{c(x): x \in B_{r}\right\} .
$$

Assume that the integral $\int d r / c(r)$ diverges. (This is certainly true if $c(r)$ is constant, as it is for the usual Dirac operator in physics.) Then it was shown in [2, Theorems 1.3 and 2.2] that $D$, with domain $C_{0}^{\infty}(\xi)$, is essentially self-adjoint. Moreover, if $f \in C^{\infty}(\xi)$ is given, then the differential equation

$$
\frac{d u}{d t}=i D u(t), u(0)=f
$$

has a global solution (namely $u(t)=e^{i t \bar{L}} f$ ) with $u(t) \in C_{0}^{\infty}(\xi)$ for all $t$. Moreover influence in (1) propagates no faster than the "speed of light." That is, if supp $f \subseteq B_{r} \subseteq B_{R}$, and $c=c(R)$, then supp $u(t) \subseteq$ $B_{r+c(t)}$ so long as $r+c(t) \leqq R$.

We will deal with perturbations of $D$ by zeroth-order operators $V$. Such an operator maps sections of $\xi$ to sections of $\xi$ and is of the form $(V f)(x)=V(x) \cdot f(x)$ where $V(x) \in \operatorname{Hom}\left(\xi_{x}, \xi_{x}\right)$; in local coordinates, $V$ is a matrix-valued function. We require that $V(x)$ be symmetric for all $x$, and that $x \mapsto V(x)$ be measurable. We say that $V$ is locally $p$ th power integrable, and write $V \in L_{\text {loc }}^{p}$, provided that $\|V(x)\|^{p}$ is locally integrable.

Now we introduct three classes of perturbing potentials $V$ :

1. $\mathscr{C}(D)$ is the class of all " $D$-good" potentials $V: V \in L_{\text {loc }}^{2}$ and $D+V$ is essentially self-adjoint on $C_{0}^{\infty}(\xi)$. (Note. we require that $V \in L_{\text {loc }}^{2}$ in order that $C_{0}^{\infty}(\xi)$ be contained in the domain of $V$ as an operator on $L^{2}(\xi)$.)

2. $\mathscr{V}_{\text {loc }}(D)$ is the class of all $V$ such that for each $p \in M$, there exists a member $V_{p} \in \mathscr{V}(D)$ with $V(x) \equiv V_{p}(x)$ on some neighborhood of $p$.

3. $\mathscr{V}_{1 \mathrm{loc}^{*}}(D)$ is the class of all $V$ such that, for every compact 
$K \cong M$, there is a $V_{K} \in \mathscr{V}(D)$ with $V \equiv V_{p}$ on some neighborhood of $K$. Obviously $\mathscr{V}(D) \subseteq \mathscr{\mathscr { V }}_{1 \mathrm{o} * *}(D) \subseteq \mathscr{V}_{\mathrm{loc}}(D)$. Our main result says that in fact these classes coincide.

THEOREM 2.1. With the above definitions, $\mathscr{Y}(D)=\mathscr{V}_{\text {loc }}(D)$. (That is, essential self-adjointness of $D+V$ depends only on the local behavior of $V$.)

Proof. Let $V \in \mathscr{V}_{\mathrm{loc}}(D)$ be given. We write $L$ for $D+V$ with domain $\mathscr{D}(L)=C_{0}^{\infty}(\xi)$, and $\bar{L}$ for the closure of $L$. Then $L \subseteq \bar{L} \leqq L^{*}$; $\bar{L}$ is symmetric. We shall show that $\bar{L}$ is self-adjoint.

Lemma 2.2. If $u \in \mathscr{D}(\bar{L})$ and $\phi \in C_{0}^{\infty}(M)$, then $\phi \cdot u \in \mathscr{D}(\bar{L})$; similarly, if $u \in \mathscr{D}\left(L^{*}\right)$ then $\phi \cdot u \in \mathscr{D}\left(L^{*}\right)$.

Proof. Suppose that $u \in \mathscr{D}\left(L^{*}\right)$. This means that $u \in L^{2}(\xi)$ and that as a distribution $D u+V u=L^{*} u \in L^{2}(\xi)$. Now

$$
\begin{aligned}
(D+V)(\phi u) & =D(\phi u)+\phi V u \\
& =\phi D u+\left(D_{1} \phi\right) \cdot u+\phi V u ;
\end{aligned}
$$

that is,

$$
(D+V)(\phi u)=\phi L^{*} u+\left(D_{1} \phi\right) \cdot u
$$

where $D_{1}$ is a certain first-order differential operator whose coefficients are related to those of $D$. The right side of (2) is clearly a member of $L^{2}(\xi)$, and hence $\phi \cdot u \in \mathscr{D}\left(L^{*}\right)$.

If in addition $u \in \mathscr{D}(\bar{L})$, then there is a sequence $\left\{u_{n}\right\}_{1}^{\infty}$ in $\mathscr{D}(L)$ with $u_{n} \rightarrow u$ and $L u_{n} \rightarrow \bar{L} u$. Then clearly $\phi \cdot u_{n} \in \mathscr{D}(L), \phi \cdot u_{n} \rightarrow \phi \cdot u$, and

$$
L\left(\phi \cdot u_{n}\right)=\phi L\left(u_{n}\right)+\left(D_{1} \phi\right) \cdot u_{n} .
$$

The right side of (3) converges to $\phi \bar{L} u+\left(D_{1} \phi\right) \cdot u=L^{*}(\phi u) \in L^{2}(\xi)$. Hence, by the definition of the closure of an operator, $\phi \cdot u \in \mathscr{D}(\bar{L})$ and we have the formula $\bar{L}(\phi u)=\phi \bar{L} u=\left(D_{1} \phi\right) \cdot u$.

Note that we used only the local square-integrability of $V$ in the proof.

COROLLARY 2.3. If $u \in \mathscr{D}(\bar{L})$ has compact support contained in an open set $\Omega$, then there is a sequence $\left\{u_{n}\right\}_{1}^{\infty}$ in $\mathscr{D}(L)$ with supp $u_{n} \leqq \Omega$ such that $u_{n} \rightarrow u$ and $L u_{n} \rightarrow \bar{L} u$.

Proof. Choose a $C^{\infty}$ function $\phi$ with support in $\Omega$ and $\phi \equiv 1$ on a neighborhood of the support of $u$. As in the proof of Lemma 2.2, 
take a sequence $\left\{v_{n}\right\}_{1}^{\infty}$ in $\mathscr{D}(L)$ with $v_{n} \rightarrow u$ and $L v_{n} \rightarrow \bar{L} u$. Finally, let $u_{n}=\phi \cdot v_{n}$. Then $u_{n} \in \mathscr{D}(L)$, supp $u_{n} \subseteq \Omega$, and the proof of Lemma 2.2 shows that $u_{n} \rightarrow \phi \cdot u=u$ while $L u_{n} \rightarrow \bar{L}(\phi \cdot u)=\bar{L} u$.

Lemma 2.4. Suppose $W \in \mathscr{V}(D)$. Define $N=\overline{D+W}$. Then if $f \in \mathscr{D}(N)$ and $f$ has compact support, the differential equation

$$
\frac{d u}{d t}=i N u(t), \quad u(0)=f
$$

has a unique solution, namely $u(t)=e^{i t N} f$. Moreover for each $t, u(t)$ has compact support; if supp $f \subseteq B_{r} \subseteq B_{R}$ and $c=c(R)$ then $u(t)$ is supported in $B_{r+c(t)}$ for $r+c(t) \leqq R$.

Proof. All the conclusions are immediate except for the assertion about the support of $u(t)$. For the latter we consider the Trotter product formula:

$$
u(t)=\lim _{n \rightarrow \infty}\left(e^{i t \bar{D} / n} e^{i t W / n}\right)^{n} f .
$$

Because $f$ is supported in $B_{r}$ and $e^{i t W / n}$ is merely a multiplication operator, it follows that $e^{i t W / n} f$ is supported in $B_{r}$. But then $e^{i t \bar{D} / n} e^{i t W / n} f$ is supported in $B_{r+c|t| / n}$. Iterating this process $n$ times, we see that $\left(e^{i t \bar{D} / n} e^{i t W / n}\right)^{n} f$ is supported in $B_{r+n c|t| / n}=B_{r+c|t|}$. Hence so is the limit vector $u(t)$.

We come next to the heart of argument: The proof of an analogue of Lemma 2.4 for the given potential $V \in \mathscr{Y}_{\text {loc }}(D)$.

LEMMA 2.5. Fix $V \in \mathscr{V}_{\text {loc }}(D)$. Let $f$ be a compactly supported member of $\mathscr{D}(\bar{L})=\mathscr{D}(\overline{D+V)}$. Then the differential equation

$$
\frac{d u}{d t}=i \bar{L} u(t), \quad u(0)=f
$$

has a unique global solution $u(t),-\infty<t<\infty$. Moreover $u(t)$ is compactly supported for all $t$.

Proof. The uniqueness follows in the usual way from the symmetry of $\bar{L}$.

For the existence proof, we suppose that $f$ is supported in $B_{r}$. We shall show that there exists a solution of $(6)$ for $0 \leqq t \leqq 1 / c(r+2)$ with support in $B_{r+1}$. Proceeding inductively, we can then construct a solution for all $t \geqq 0$ because the series $1 / c(r+2)+1 / c(r+3)+\cdots$ is divergent. Similarly we get a solution for all negative $t$.

We turn to the first step in the construction. Since $B_{r+2}$ is com- 
pact (by the completeness of $M$ ) and $V \in \mathscr{\mathscr { l }} \mathscr{L}_{\mathrm{loc}}(D)$ we may find a positive number $2 \varepsilon$ with the following property: for each $p \in B_{r+2}$, if $\Omega_{p, 2 \varepsilon}$ is the $2 \varepsilon$-ball with center $p$, there is a $V_{p} \in \mathscr{V}(D)$ which coincides with $V$ on $\Omega_{p, 2 \varepsilon}$. By compactness a finite number of the corresponding $\varepsilon$-balls $\left\{\Omega_{p, \varepsilon}\right\}_{1}^{m}$ cover $B_{r+2}$.

Let $\left\{\theta_{p}\right\}_{1}^{m}$ be a corresponding set of $C_{0}^{\infty}$ functions with $\theta_{p}$ supported in $\Omega_{p, \varepsilon}$ and $\sum_{p=1}^{m} \theta_{p} \equiv 1$ on $B_{r+1}$.

Returning to the initial vector $f$, define $f_{p}=\theta_{p} \cdot f$ for $1 \leqq p \leqq m$. Note that $f_{p}$ is supported in $\Omega_{p, \varepsilon}$ and that $f_{p} \in \mathscr{D}(\bar{L})$ by Lemma 2.2. Moreover, if we define $L_{p}=D+V_{p}$, we have the relations (in the sense of distributions)

$$
\bar{L} f_{p}=D f_{p}+V f_{p}=D f_{p}+V_{p} f_{p}=L_{p}^{*} f_{p}=\bar{L}_{p} f_{p},
$$

where the last equality follows from the self-adjointness of $\bar{L}_{p}$. In particular, $f_{p} \in \mathscr{D}\left(\bar{L}_{p}\right)$.

Now let $\tau=\varepsilon / c(r+2)$. For $0 \leqq t \leqq \tau$, define

$$
u(t)=\sum_{p=1}^{m} e^{i t L p} f_{p}=\sum_{p=1}^{m} u_{p}(t) .
$$

Then $u(0)=\sum f_{p}=f, u(t)$ is differentiable, and we have the formula

$$
\frac{d}{d t} u(t)=\sum_{p=1}^{m} i \bar{L}_{p} u_{p}(t) \text {. }
$$

Moreover, Lemma 2.4 implies that $u_{p}(t)$ is supported in $\Omega_{p, 2 \varepsilon}$ for $0 \leqq$ $t \leqq \tau$. It then follows from Corollary 2.3 that $\bar{L}_{p} u_{p}(t)=\bar{L} u_{p}(t)$. Reason: because $u_{p}(t)$ is supported in $\Omega_{p, 2 \varepsilon}$, there exist $C^{\infty}$ functions $v_{n}$ supported in $\Omega_{p, 2 \varepsilon}$ with $v_{n} \rightarrow u_{p}(t)$ and $L_{p} v_{n} \rightarrow \bar{L}_{p} u_{p}(t)$. But since $v_{n}$ is supported in $\Omega_{p, 2 \varepsilon}$ we have $L_{p} v_{n}=L v_{n}$. We can conclude that $u_{p}(t) \in$ $\mathscr{D}(\bar{L})$ and that $\bar{L} u_{p}(t)=\bar{L}_{p} u_{p}(t)$.

Thus, from (7), we have, for $0 \leqq t \leqq \tau$,

$$
\frac{d}{d t} u(t)=\sum_{p=1}^{m} i \bar{L} u_{p}(t)=i \bar{L} \sum_{p=1}^{m} u_{p}(t)=i \bar{L} u_{p}(t) \text {. }
$$

That is, the differential equation (6) is satisfied, $0 \leqq t \leqq \tau$. Furthermore, $u(\tau) \in \mathscr{D}(\bar{L})$ and is obviously supported in $B_{r+c \tau}, c=c(r+2)$. This is so because, for all $p, f_{p}$ is supported in $B_{r}$; hence by Lemma 2.4 (with $\left.W=V_{p}\right) u_{p}(t)$ is supported in $B_{r+c t}, 0 \leqq t \leqq \tau$. And so $u(t)=$ $\sum u_{p}(t)$ is supported in $B_{r+c t}$.

Now we start the process over again, assuming that $r+c \tau<$ $r+1$, with $u(\tau)$ as the new initial data. We thus extend the solution $u(t)$ throughout the interval $\tau \leqq t \leqq 2 \tau$. This may be continued for $n$ steps, until $r+n c \tau \geqq r+1$ (the final step is modified by shortening 
$\tau$ if necessary so as not to overshoot the mark). Of course the crucial fact that makes this argument work is that $\tau$ does not depend on the initial data.

Completion of the proof of Theorem 2.1. We can now deduce that $\bar{L}$ is self-adjoint by means of the "invariance of domain" method (cf. [2, Lemma 2.1]). Let $\mathscr{D}$ consist of the compactly supported members of $\mathscr{D}(\bar{L})$. We have just shown that for every $f \in \mathscr{D}$ there is a solution $u(t)$ of the differential equation (6) with $u(0)=f$ and $u(t) \in \mathscr{D}$ for all $t$. Moreover $\|u(t)\|^{2}$ is constant because $\bar{L}$ is symmetric.

Denote by $A$ the restriction of $\bar{L}$ to $\mathscr{D}$. Suppose that $A^{*} \psi=$ $\pm i \psi$. We claim that $\psi$ must be 0 . (This will establish that $A$ is essentially self-adjoint and hence that $\bar{L}$ is self-adjoint.) Consider the case $A^{*} \psi=i \psi$. Given $f \in \mathscr{D}$ let $u(t)$ be the corresponding solution of (6). Define

$$
F(t)=(u(t), \psi)
$$

Then $F(t)$ is bounded, $-\infty<t<\infty$, and we have

$$
F^{\prime}(t)=(i \bar{L} u(t), \psi)=\left(i u(t), A^{*} \psi\right)=(i u(t), i \psi)=F(t) .
$$

Hence $F(t)=F(0) e^{t}$. Since $F$ is bounded we must have $F(0)=0$; that is, $(f, \psi)=0$. Since $\mathscr{D}$ is dense, $\psi$ must be 0 . A similar argument takes care of the case: $A^{*} \psi=-i \psi$.

Since Theorem 2.1 shows that only the local behavior of $V$ affects the essential self-adjoitness of $D+V$, it follows immediately that if $V \in \mathscr{V}(D)$ and $W$ is locally bounded, then $V+W \in \mathscr{Y}(D)$. For explicit local conditions that guarantee that $V$ belongs to $\mathscr{V}(D)$ see [5] or [6].

3. Abstract wave equations. In this section we collect some results on second-order operator differential equations which we will later apply to study Schrödinger operators by means of the allied wave equations.

We begin by recalling some standard facts. If $S \geqq a>0$ is a semibounded self-adjoint operator on a Hilbert space $\mathscr{H}$, we can solve the second-order equation $d^{2} u / d t^{2}=-S u(t)$ by transforming it into a first-order system: $d u / d t=v, d v / d t=-S u$. More precisely, we form the Hilbert space $\boldsymbol{H}=\mathscr{D}\left(\boldsymbol{S}^{1 / 2}\right) \oplus \mathscr{H}$, with norm $\|(u, v)\|^{2}=$ $\left\|S^{1 / 2} u\right\|^{2}+\|v\|^{2}$, and the operator

$$
A=\left[\begin{array}{rr}
0 & I \\
-S & 0
\end{array}\right]
$$


with domain $\mathscr{D}(A)=\mathscr{D}(S) \oplus \mathscr{D}\left(S^{1 / 2}\right)$. Then $A$ is a skew-adjoint operator on $\boldsymbol{H}$ and generates the one-parameter unitary group

$$
e^{t A}=\left[\begin{array}{lc}
\cos t \sqrt{S} & \frac{\sin t \sqrt{S}}{\sqrt{S}} \\
-\sqrt{S} \sin t \sqrt{S} & \cos t \sqrt{S}
\end{array}\right]
$$

If $\psi(t)=e^{t A} \psi(0)$ has components $(u(t), v(t))$ then $u(t)=\cos t \sqrt{S} f+$ $\sin t \sqrt{S} / \sqrt{S} g$, where $f=u(0), g=v(0)$; if $f \in \mathscr{D}(S)$ and $g \in \mathscr{D}\left(S^{1 / 2}\right)$ then $u(t)$ satisfies the second-order equation $\ddot{u}(t)=-S u(t)$ with initial data $u(0)=f, \dot{u}(0)=g$.

More generally, for any real number $\alpha$, let $\mathscr{H}_{\alpha}$ be the completion of $\mathscr{H}$ in the norm $\left\|S^{\alpha} f\right\|$. Note that $\mathscr{H}_{1 / 2}$ is just $\mathscr{D}\left(S^{1 / 2}\right)$ with the graph norm. We have the inclusions

$$
\mathscr{H}_{1 / 2} \subseteq \mathscr{H} \subseteq \mathscr{H}_{-1 / 2},
$$

and $S$ extends by continuity to an isometry of $\mathscr{H}_{1 / 2}$ with $\mathscr{H}_{-1 / 2}$. Let $\boldsymbol{K}$ be the Hilbert space $\mathscr{H} \oplus \mathscr{H}_{-1 / 2}$. Then the matrix formula (1) defines a skew-adjoint operator $A_{1}$ on $K$, with $\mathscr{D}\left(A_{1}\right)=\mathscr{D}\left(S^{1 / 2}\right) \oplus \mathscr{H}$, and the group generated by $A_{1}$ is given by formula (2). This alternative conversion of the second-order equation into a first-order system will be useful later for technical reasons.

Now let $C$ be another operator on $\mathscr{H}$ which is bounded relative to $S$ with relative bound less than 1: that is, $\mathscr{D}(S) \subseteq \mathscr{D}(C)$ and there are constants $\gamma<1$ and $k<\infty$ with $\|C f\| \leqq \gamma\|S f\|+k\|f\|$ for all $f \in \mathscr{D}(S)$. Consider the differential equation

$$
\ddot{u}(t)=-S u(t)-C u(t) \text {. }
$$

We write this as a first-order system on $\boldsymbol{H}=\mathscr{D}\left(\boldsymbol{S}^{1 / 2}\right) \oplus \mathscr{H}$ as follows:

$$
\begin{aligned}
& \dot{u}=v \\
& \dot{v}=-S u-C u .
\end{aligned}
$$

That is, if $\psi=(u, v)$,

$$
\frac{d \psi}{d t}=A \psi+B \psi
$$

where $A$ is defined by (1) and $B=\left[\begin{array}{rr}0 & 0 \\ -C & 0\end{array}\right]$. Unfortunately $B$ is a rather bad perturbation of $A$ even though $C$ is a good perturbation of $S$; for instance, $B$ does not generate a semigroup on $\boldsymbol{H}$. Nevertheless, the following result is true. 
$\mathscr{H}$. Let $C$ be a closed symmetric operator on $\mathscr{H}$ which is bounded relative to $S$ with relative bound less than 1 . Then the operator $L$ defined by the relation

$$
L=\left[\begin{array}{cc}
0 & 0 \\
-S-C & I
\end{array}\right]
$$

with domain $\mathscr{D}(L)=\mathscr{D}(S) \oplus \mathscr{D}\left(S^{1 / 2}\right)$, generates a $\left(C_{0}\right)$ group on $\boldsymbol{H}=\mathscr{D}\left(\boldsymbol{S}^{1 / 2}\right) \oplus \mathscr{H}$.

Proof. First, note that by the Kato-Rellich theorem the operator $T=S+C$ is self-adjoint with domain $\mathscr{D}(T)=\mathscr{D}(S)$. In addition, $T$ is semibounded. Indeed, since $C$ has relative bound $\leqq \gamma<1$, there is a constant $k$ such that

$$
\|C f\| \leqq \gamma\|(S+k) f\|
$$

for all $f \in \mathscr{D}(S)$. Hence, by the monotonicity of the square root on operators (cf. [7, Th. V. 4.12] we have

$$
(|C| f, f) \leqq \gamma((S+k) f, f)
$$

for all $f \in \mathscr{D}(S)$. Hence we have the inequalities

$$
S+C \geqq S-|C|=(1-\gamma) S+\gamma S-|C| \geqq-\gamma k .
$$

Also, the operator $\left[\begin{array}{ll}0 & 0 \\ \alpha & 0\end{array}\right]$, where $\alpha$ is any constant, is a bounded perturbation on $\boldsymbol{H}$. Accordingly, by adding a suitable constant $\alpha \geqq$ $1+\gamma k$ to $C$, we may arrange that $S+C$ be $\geqq 1$ without affecting the issue of whether $L$ is a generator.

But then our preliminary remarks show that equation (3) $(\ddot{u}=-T u)$ can be solved on the space $\mathscr{D}\left(T^{1 / 2}\right) \oplus \mathscr{H}$, and that $L$ is a generator on this space with domain $\mathscr{D}(T) \oplus \mathscr{D}\left(T^{1 / 2}\right)$. So all we have to do is prove that $\mathscr{D}\left(T^{1 / 2}\right) \oplus \mathscr{H}$ coincides with $\boldsymbol{H}$-i.e., that $\mathscr{D}\left(T^{1 / 2}\right)=$ $\mathscr{D}\left(S^{1 / 2}\right)$. (The graph norms will then automatically be equivalent, by the closed graph theorem.)

Since $\mathscr{D}(S)=\mathscr{D}(T)$ and $T \geqq 1$, there is a constant $\delta$ such that, for all $f \in \mathscr{D}(T)=\mathscr{D}(S)$,

$$
\|S f\| \leqq \delta\|T f\|
$$

Then by another application of [7, Th. V. 4.12] we have

$$
(S f, f) \leqq \delta(T f, f)
$$

for all such vectors $f$. Similarly there is a constant $\delta^{\prime}$ such that

$$
(T f, f) \leqq \delta^{\prime}(S f, f) \text {. }
$$


Hence the two inner products $(S f, g)$ and $(T f, g)$ are equivalent on $\mathscr{D}(S)=\mathscr{D}(T)$. But $\mathscr{D}\left(S^{1 / 2}\right)$ is just the completion with respect to the first inner product, while $\mathscr{D}\left(T^{1 / 2}\right)$ is the completion with respect to the second inner product. This proves that $\mathscr{D}\left(S^{1 / 2}\right)=\mathscr{D}\left(T^{1 / 2}\right)$.

We also have a result about approximation of solutions to (3).

CoRollary 3.2. Let $S$ and $C$ be operators as in Proposition 3.1. Define $\chi_{n}(\lambda)=\lambda$ if $|\lambda| \leqq n,=0$ otherwise; and consider the "cutoff" perturbations $C_{n}=\chi_{n}(C)$.

On $\boldsymbol{H}=\mathscr{D}\left(\boldsymbol{S}^{1 / 2}\right) \oplus \mathscr{H}$, let $e^{t L_{n}}$ be the $\left(C_{0}\right)$ group generated by the operator

$$
L_{n}=\left[\begin{array}{cc}
0 & 1 \\
-S-C_{n} & 0
\end{array}\right]
$$

and let $L$ be as in equation (4). Then as $n \rightarrow \infty$

$$
e^{t L_{n}} \longrightarrow e^{t L}
$$

in the strong operator topology on $\boldsymbol{H}$.

Proof. Since $\left\|C_{n} f\right\| \leqq\|C f\|$ for all $f \in \mathscr{D}(S)$, the estimates (5), (6), and (7) of Proposition 3.1 all hold with $C_{n}$ replacing $C$. Thus if we add the constant $\alpha=\gamma k+1$ to all $C_{n}$ 's and $C$, we may assume without loss of generality that $S+C \geqq 1$ and $S+C_{n} \geqq 1$ for all $n$.

Write $T=S+C$ and $T_{n}=S+C_{n}$. Then $L=\left[\begin{array}{rr}0 & I \\ -T & 0\end{array}\right]$, and $L_{n}=\left[\begin{array}{ll}0 & I \\ T_{n} & 0\end{array}\right]$. By Proposition 3.1, we know that $\mathscr{D}\left(L_{n}\right)=\mathscr{D}(L)=$ $\mathscr{D}(S) \oplus \mathscr{D}\left(S^{1 / 2}\right)$. Moreover it is trivial that $L_{n} \psi \rightarrow L_{\psi} \psi$ for any $\psi \in \mathscr{D}(L)$. Hence the Trotter-Kato theorem [7, Th. IX. 2.16] will show that $e^{t L_{n}} \rightarrow e^{t L}$ provided that we verify that these groups are uniformly bounded on $\boldsymbol{H}$ with a bound independent of $n$.

We know that $e^{t L_{n}}$ is a group of isometries relative to the norm induced from $\mathscr{D}\left(T_{n}^{1 / 2}\right) \oplus \mathscr{X}$. We denote this norm by $\|\cdot\|_{n}$, while we denote the norm from $\mathscr{D}\left(S^{1 / 2}\right) \oplus \mathscr{H}$ by $\|\cdot\|$. The proof will be finished if we can show that there are constants $\alpha, \beta$ in $(0, \infty)$ such that

$$
\alpha\|\cdot\| \leqq\|\cdot\|_{n} \leqq \beta\|\cdot\| ;
$$

for then we will have the estimate $\left\|e^{t L_{n}}\right\| \leqq \beta / \alpha$ for all real $t$ and all $n=1,2, \cdots$.

Because of our normalization, it follows from (7), with $C$ replaced by $C_{n}$, that

$$
T_{n}=S+C_{n} \geqq(1-\gamma) S+1
$$


Similarly, if $\lambda$ is large enough, we have

$$
C_{n} \leqq \lambda S
$$

for all $n$. Hence there are constants $\alpha, \beta$ independent of $n$ such that

$$
\alpha^{2} S \leqq T_{n} \leqq \beta^{2} S \text {. }
$$

Now (8) is an immediate consequence of (9).

We turn next to some simple facts about time-dependent equations.

Proposition 3.3. Let $e^{t A}$ be a $\left(C_{0}\right)$ group on the Hilbert space $\boldsymbol{H}$, and let $B(t)$ be a norm-continuous function from the reals to the bounded operators on $\boldsymbol{H}$. Assume also that, for each $t$, the operator $B(t)$ maps $\mathscr{D}(A)$ into itself, and that $t \mapsto B(t)$ is continuous as a map from the reals into the bounded operators on $\mathscr{D}(A)$ (equipped with the graph norm).

Then, for each $f \in \mathscr{D}(A)$, the differential equation

$$
\frac{d \psi}{d t}=A \psi(t)+B(t) \psi(t), \psi(0)=f
$$

has a unique solution $\psi(t)$.

Proof. If $\psi(t)$ satisfies (10) then it satisfies the integral equation

$$
\psi(t)=e^{t A} f+\int_{0}^{t} e^{(t-\tau) A} B(\tau) \psi(\tau) d \tau
$$

Uniqueness of solutions to (11) follows in the usual way (Gronwall's inequality). As for existence: The additional hypothesis on $B(t)$ shows that (11) makes sense as an integral equation on $\mathscr{D}(A)$, and the usual iteration method shows that it has a solution $\psi(t) \in \mathscr{D}(A)$.

Since $\psi(t)$ satisfies $(11)$ and $\psi(\tau) \in \mathscr{D}(A)$, we have $B(\tau) \psi(\tau) \in \mathscr{D}(A)$ and so we may differentiate under the integral sign in (11) to deduce that $\psi(t)$ is a solution of the original differential equation (10).

The next proposition deals with second-order equations.

Proposition 3.4. Suppose now that $S$ is a semibounded selfadjoint operator on a Hilbert space $\mathscr{H}$, and that $\beta(t)$ is a differentiable real-valued function on the real line. Consider the time-dependent wave equation

$$
\begin{aligned}
d^{2} u / d t^{2} & =-S u(t)-\beta(t) u(t) \\
u(0) & =f \in \mathscr{D}(S), \dot{u}(0)=0 .
\end{aligned}
$$


This equation has a global solution $u(t)$. Moreover we have the "energy" formula

$$
\frac{d}{d t}\left\{\|\dot{u}(t)\|^{2}+((S+\beta(t)) u(t), u(t))\right\}=\beta^{\prime}(t)\|u(t)\|^{2} .
$$

Proof. By adding a constant to $S$ and subtracting it from $\beta(t)$, we may assume that $S \geqq 1$. Then we know that the operator $A=$ $\left[\begin{array}{rr}0 & 1 \\ -S & 0\end{array}\right]$ is skew-adjonit on the Hilbert space $\boldsymbol{H}=\mathscr{D}\left(S^{1 / 2}\right) \oplus \mathscr{H}$, with domain $\mathscr{D}(A)=\mathscr{D}(S) \oplus \mathscr{D}\left(S^{1 / 2}\right) . \quad A$ generates a $\left(C_{0}\right)$ group of operators $e^{t A}$ on $\boldsymbol{H}$. Next, consider the operator $B(t)$ on $\boldsymbol{H}$ defined by

$$
B(t)=\left[\begin{array}{cc}
0 & 0 \\
-\beta(t) & 0
\end{array}\right] \text {. }
$$

Obviously $t \mapsto B(t)$ is continuous in operator norm. Moreover, it is straightforward to verify that $B(t)$ maps $\mathscr{D}(A)$ into itself and $t \rightarrow$ $B(t)$ is norm-continuous on the space $\mathscr{D}(A)$.

By Proposition 3.3 it follows that the equation

$$
\frac{d \psi}{d t}=A \psi+B(t) \psi, \quad \psi(0)=\left[\begin{array}{l}
f \\
0
\end{array}\right]
$$

has a unique solution. If we write $\psi(t)=\left[\begin{array}{c}u(t) \\ \dot{u}(t)\end{array}\right]$ then $u(t)$ satisfies the wave equation (10).

Moreover, we have the relation

$$
\|\dot{u}(t)\|^{2}+(S u(t), u(t))=\|\psi(t)\|^{2}
$$

by the definition of the norm in $\boldsymbol{H}$. Hence

$$
\begin{aligned}
& \frac{d}{d t}\left\{\|\dot{u}(t)\|^{2}+((S+\beta(t)) u(t), u(t))\right\} \\
& \quad=\frac{d}{d t}\left\{\|\psi(t)\|^{2}+\beta(t)\|u(t)\|^{2}\right\} \\
& \quad=2 \operatorname{Re}(\psi, \dot{\psi})+2 \beta(t) \operatorname{Re}(u, \dot{u})+\beta^{\prime}(t)\|u(t)\|^{2} .
\end{aligned}
$$

From the differential equation (14), the formula $\psi=\left[\begin{array}{c}u \\ \dot{u}\end{array}\right]$, and the definition of the inner product in $\boldsymbol{H}$, we get the relation

$$
\begin{aligned}
(\dot{\psi}, \dot{\psi}) & =\left(S^{1 / 2} u, S^{1 / 2} \dot{u}\right)+(\dot{u},-S u-\beta(t) u) \\
& =\left(S^{1 / 2} u, S^{1 / 2} \dot{u}\right)-\left(S^{1 / 2} \dot{u}, S^{1 / 2} u\right)-\beta(t)(\dot{u}, u) .
\end{aligned}
$$

Here we have used the fact that $\dot{u}(t) \in \mathscr{D}\left(S^{1 / 2}\right)$. It follows that $\operatorname{Re}(\psi, \dot{\psi})=-\beta(t) \operatorname{Re}(\dot{u}, u)$, so that the right-hand side of (15) reduces 
to $\beta^{\prime}(t)\|u(t)\|^{2}$. That is, equation (13) holds.

Finally, we have a strong uniqueness theorem.

Proposition 3.5. Let $T$ be a semibounded symmetric operator on $\mathscr{H}$. Let $\beta(t)$ be a continuous real-valued function on the real line. Suppose that $u(t), 0 \leqq t \leqq t_{0}$, is a solution of the differential equation

$$
\begin{aligned}
d^{2} u / d t^{2} & =-T u(f)-\beta(t) u(t) \\
u(0) & =\dot{u}(0)=0 .
\end{aligned}
$$

Then $u(t)$ is identically 0 .

Proof. As in Proposition 3.4, we may assume without loss of generality that $T \geqq 1$. Let $S$ be the Friedrichs extension of $T$. Form the Hilbert space $\boldsymbol{K}=\mathscr{H} \oplus \mathscr{H}_{-1 / 2}$ as discussed in the third paragraph of this section, and consider on $K$ the operator $A_{1}=\left[\begin{array}{rr}0 & I \\ -S & 0\end{array}\right]$ and the corresponding one-parameter group $e^{t A_{1}}$. Also define an operator $B_{1}(t)$ on $K$ by the formula

$$
B_{1}(t)=\left[\begin{array}{cc}
0 & 0 \\
-\beta(t) & 0
\end{array}\right]
$$

Then $t \mapsto B_{1}(t)$ is a norm-continuous operator-valued function on $\boldsymbol{K}$.

Consider the curve $\psi(t)=\left[\begin{array}{l}u(t) \\ \dot{u}(t)\end{array}\right]$. It is easy to see that $\psi(t)$ is a differentiable curve in $\boldsymbol{K}$ which satisfies the differential equation

$$
\dot{\psi}(t)=A_{1} \psi(t)+B(t) \psi(t), \quad \psi(0)=0 .
$$

The usual uniqueness proof (reduction to an integral equation plus Gronwall's inequality) then shows that $\psi(t)$ is identically 0 .

Note. In Proposition 3.5 it was necessary to introduce the space $K$ because it is not evident a priori that $\psi(t)$ is differentiable as a curve in the space $\boldsymbol{H}=\left(\boldsymbol{S}^{1 / 2}\right) \oplus \mathscr{H}$.

4. Schrödinger operators. We begin by recalling some of the results of [2] concerning second-order operators with smooth coefficients. Suppose that $M$ is a complete Riemannian manifold and that $\Lambda$ is a symmetric, negative, second-order elliptic operator on $M$. Let $c(x)$ denote the local propagation speed associated with $\Lambda$; roughly, $c(x)$ is the square root of the largest eigenvalue of the symbol of $\Lambda$. For example, if $\Lambda=\Delta$, the Laplace-Beltrami operator, then $c(x)$ is identically 
1. Fix a point $0 \in M$, and let $B_{r}$ denote the ball of radius $r$ and center 0 . Note that $\bar{B}_{r}$ is compact because $M$ is complete. Define $c(r)=\sup \left\{c(x): x \in B_{r}\right\}$. As with the Dirac-type operators discussed in $\S 2$, we assume that the integral $\int^{\infty} d r / c(r)$ diverges. Then it was shown in [2] that $\Lambda$, with domain $C_{0}^{\infty}(M)$, is essentially self-adjoint. Moreover-the point of greatest interest here-the wave equation $\ddot{u}(t)=\Lambda u(t)$ has the following propagation property: if the initial data $u(0), \dot{u}(0)$ have compact support then so does $u(t)$ for all $t$.

We shall state a fairly precise form of this result, which says that data supported in $B_{r}$ propagates with instantaneous velocity at most $c(r)$. For $r \geqq 0$ we define $\theta(r)=\int_{0}^{r} d s / c(s)$. The assumption about the divergence of the integral to $\infty$ shows that $\theta$ is a diffeomorphism of $[0, \infty)$ with itself. Moreover, if $R<r$ then $\theta(r)-\theta(R)=t$ gives a lower bound for the time during which the influence of data supported in $B_{R}$ remains inside $B_{r}$. Hence, given $t>0$ and $R$, if $u(0)$ and $\dot{u}(0)$ are supported in $B_{R}$ then $u(t)$ will be supported in $B_{r}$, where $r=\theta^{-1}(t+\theta(R))$. Let us write $t * R$ for $\theta^{-1}(t+\theta(R))$. Thus data supported in $B_{R}$ generates a solution supported at time $t$ within $B_{t * R}$. (Note the identity $t_{1} *\left(t_{2} * R\right)=\left(t_{1}+t_{2}\right) * R$, whose interpretation in terms of support propagation is obvious.)

We are concerned with the Schrödinger equation $1 / i(\partial u / \partial t)=$ $-A u+V u$, where $V$ is a potential on $M$ which may be rather singular. We assume henceforth that in local coordinates $\Lambda$ has the form $A u=$ $\sum_{i j} \partial_{i}\left(a_{i j} \partial_{j} u\right)$, i.e., $\Lambda u=\operatorname{div}(a \operatorname{grad} u)$, where $a$ is a smooth matrixvalued function. (This means that we are neglecting "magnetic fields" which would appear as lower-order lerms in 1 . Actually, at the cost of some technical complication, our arguments could cope with arbitrary smooth magnetic fields, but we shall stick to the simpler case in writing out the proofs.) We seek conditions on $V$ which guarantee that the operator $-\Lambda+V$ is essentially self-adjoint on the domain $C_{0}^{\infty}(M)$ We shall use the strategy of [2]; namely, we shall relate the self-adjointness question for the operator $T=-\Lambda+V$ to that of existence of solutions to the wave equation $\ddot{u}(t)=-T u(t)$. (A related approach has been explored by Berezanskii [1], who relies upon a uniqueness theorem for the adjoint wave equation $\ddot{u}(t)=$ $-T^{*} u(t)$ rather than an existence theorem for the original equation. Our method seems to yield stronger results.) We will impose local conditions on the potential $V$ to guarantee that $-\Lambda+V$ is "locally" essentially self-adjoint, together with a global semiboundedness condition, of the form $-\Lambda+V \geqq-\left(a+b \theta(r)^{2}\right)$, which is the analogue of a classical condition that insures that particle trajectories do not reach infinity in a finite time. Here $r$ is the function $r(x)=d(x, 0)$, the distance to the "origin" $0 \in M$. Note that in case $\Lambda=\Delta$ we have 
$c(r)=1$ so that $\theta(r)=r$ and our condition reduces to the well known condition $-\Delta+V \geqq-\left(a+b r^{2}\right)$.

We turn first to the local problem. There are a number of techniques in the literature which we might use. We shall employ the following three known theorems, which we list for the reader's convenience.

Theorem A ((Faris) [3, Thm. 4.1], [4, Thm. 8.3]). Let $\mathscr{H}=$ $L^{2}(m, \mu)$. Let $e \in \mathscr{H}, e>0$ a.e. Let $H_{0} \geqq 0$ be a self-adjoint operator acting in $\mathscr{H}$ with $H_{0} e=0$ Assume that $\left(H_{0}+c\right)^{-1}$ is positivitypreserving for all $c>0$. Let $U \geqq 0$ be a real function in $L^{2}\left(M, e^{2} \mu\right)$.

Let $H=H_{0}+U$. Then $H$ is essentially self-adjoint on $\mathscr{D}(H)=$ $\mathscr{D}\left(H_{0}\right) \cap \mathscr{D}(U)$. Moreover $(H+c)^{-1} L_{\infty}(e)$ is a domain of essential self-adjointness (a "core") for $H$.

Theorem B ((Davies-Faris) [3, Thm. 4.4], [10, Thm. X. 31]). Let $H_{0}$ be a positive self-adjoint operator on $L^{2}(M, \mu)$ such that $\left(H_{0}+c\right)^{-1}$ is positivity-preserving. Let $U \geqq 0$ be a multiplication operator. Suppose that $H=H_{0}+U$ is essentially self-adjoint. Let $W$ be a multiplication operator which is bounded relative to $H_{0}$. Then $W$ is also H-bounded. Indeed, if we have

$$
\|W \psi\| \leqq a\left\|\left(H_{0}+b\right) \psi\right\|, \quad \psi \in \mathscr{D}\left(H_{0}\right)
$$

then we have the estimate

$$
\|W \psi\| \leqq a\|(H+b) \psi\|, \quad \psi \in \mathscr{D}(H)
$$

with the same constants $a, b$.

Theorem C. Let $M$ be compact Riemannian manifold. Let $A$ be a second-order elliptic operator on $M$. Let $d$ be the dimension of M. Following [12], we say that $p$ is "d-canonical" if $p=2$ for $d \leqq 3 ; p>2$ if $d=4 ; p=d / 2$ if $d \geqq 5$.

Suppose $p$ is d-canonical and $W \in L^{p}(M)$. Then the multiplication operator $W$ is bounded relative to $\Lambda$ with relative bound 0.

Proof. In case $\Lambda=\Delta$ on $\boldsymbol{R}^{d}$, this follows from Sobolev inequalities (cf. [10, Thms. X. 16, X. 30, X. 21]; the case $p=2, d=3$ is a famous result of Kato).

In the manifold case the conclusion follows from the "flat" case by a straightforward argument using a partition of unity to patch together inequalities in local charts.

Proposition 4.1. Let $M$ be a compact Riemannian manifold, 
dimension $d$. Let $\Lambda$ be a second-order elliptic operator on $M: \Lambda u=$ $\operatorname{div}(a \operatorname{grad} u), a$ smooth. Let $U \geqq 0$ belong to $L^{2}(M)$, and let $W \geqq 0$ belong to $L^{p}(M)$, where $p$ is d-canonical. Write $V=U-W$. Let $H=-\Lambda+V$. Then $H$ is semibounded and essentially self-adjoint on $C^{\infty}(M)$.

Proof. We shall apply Theorem A, with $H_{0}=-\bar{\Lambda}$. The first step is to check that if $c>0$ then $(-\bar{\Lambda}+c)^{-1}$ is positivity-preserving. This follows from the classical maximum principle. (Indeed, suppose that $\phi \in C^{\infty}(M), \phi \geqq 0$, and $(-\bar{\Lambda}+c) \psi=\phi$. We must show $\psi \geqq 0$. By elliptic regularity, $\psi$ is $C^{\infty}$. If $\psi$ is negative anywhere then $\psi$ attains a negative minimum at some point $p \in M$. Then $\psi(p)<0$ but $\Lambda \psi(p) \geqq 0$, so $\phi(p)=-\Lambda \psi(p)+c \psi(p)<0$, a contradiction. Thus $(-\bar{A}+c)^{-1}$ is positivity-preserving on the positive cone in $C^{\infty}(M)$, which is dense in the positive cone of $L^{2}(M)$.)

Now define $e(x) \equiv 1$. Then $e>0$ everywhere, $e \in L^{2}(M)$, and $\Lambda e=0$. Moreover $U \in L^{2}\left(M, e^{2} \mu\right)=L^{2}(M)$. Hence, by Theorem A, it follows that $-\bar{\Lambda}+U=H_{0}+U$ is essentially self-adjoint on $\mathscr{D}(\bar{\Lambda}) \cap$ $\mathscr{D}(U)$.

By Theorem $\mathrm{C}$, the multiplication operator $W$ is bounded relative to $H_{0}$ with relative bound 0 . Hence, by Theorem $\mathrm{B}, W$ is bounded relative to $H_{1}=H_{0}+U$ with relative bound 0 . It follows that $H_{1}-W=H_{0}+U-W$ is semibounded and essentially self-adjoint on $\mathscr{D}\left(H_{1}\right) \cap \mathscr{D}(W)$. Moreover any core for $H_{1}$ is a core for $H_{1}-W$.

The proof will be finished if we show that $C^{\infty}(M)$ is a core for $H_{1}$. Now, by Theorem $\mathrm{A},\left(H_{1}+c\right)^{-1} L^{\infty}(M)$ is a core for $H_{1}$ Hence the larger space $\mathscr{C}=L^{\infty}(M) \cap \mathscr{D}(-\bar{\Lambda})$ is a core for $H_{1}$. To prove that $C^{\infty}(M)$ is a core for $H_{1}$, we have to show that a given $f \in \mathscr{M}$ may be approximated by a sequence $f_{n} \in C^{\infty}(M)$ so that $H_{1} f_{n} \rightarrow H_{1} f$, at least weakly. In fact we shall construct such a sequence with $\left\|f_{n}\right\|_{\infty} \leqq\|f\|_{\infty}$ and $\Lambda f_{n} \rightarrow \bar{\Lambda} f, U f_{n} \stackrel{w}{\rightarrow} U f$.

We simply take $f_{n}=e^{\bar{\pi} n} f$. Then $\left\|f_{n}\right\|_{\infty} \leqq\|f\|_{\infty}$ by the maximum principle; $f_{n} \in C^{\infty}(M)$ by the smoothing properties of the heat equation; $f_{n} \rightarrow f$ in $L^{2}$; and $\Lambda f_{n} \rightarrow \bar{\Lambda} f$ in $L^{2}$. As for $U f_{n}$, note that $\left\|U f_{n}\right\|_{2} \leqq$ $\|U\|_{2}\left\|f_{n}\right\|_{\infty} \leqq\|U\|_{2}\|f\|_{\infty}$, so $\left\{U f_{n}\right\}_{1}^{\infty}$ is a bounded sequence in $L^{2}$. Moreover it is obvious that $U f_{n} \rightarrow U f$ in measure, so $U f$ is the unique adherent point in $L^{2}$ of the sequence $\left\{U f_{n}\right\}$. Accordingly $U f_{n} \stackrel{w}{\rightarrow} U f$.

CoRollary 4.2. Let $M$ be a compact Riemannian manifold. Let $\Lambda$ and $V$ be as in Proposition 4.1. Write $H=-\overline{\Lambda+V}$. Suppose that $f \in \mathscr{D}(H)$ has support in the ball $B_{r}$. If $s>r$ there is a sequence of $C^{\infty}$ functions $f_{n}$ with supports in $B_{s}$ such that $f_{n} \rightarrow f$ and $(-\Lambda+V) f_{n} \rightarrow H f$ in $L^{2}$. 
Proof. By Proposition 4.1, $C^{\infty}(M)$ is a core for $H$, as well as for $H_{1}=[-\Lambda+U]^{-}$; moreover, since $W$ is $H_{1}$-bounded, $\mathscr{D}\left(H_{1}\right)=\mathscr{D}(H)$. Accordingly, there is a sequence of $C^{\infty}$ functions $\left\{g_{n}\right\}_{1}^{\infty}$ such that $g_{n} \rightarrow f,(-\Lambda+U) g_{n} \rightarrow H_{1} f$, and $W g_{n} \rightarrow W f$; in particular $(-\Lambda+V) g_{n} \rightarrow$ $H f$.

Choose a $C^{\infty}$ function $\phi$ such that $\phi$ is supported in the ball $B_{s}$ and $\phi$ is identically 1 in a neighborhood of the support of $f$ : thus $\phi f=f$ and $\phi H f=H f$. Define $f_{n}=\phi g_{n}$. Clearly $f_{n}$ is $C^{\infty}$, supported in $B_{s}$, and $f_{n} \rightarrow f$. It remains to show that $(-\Lambda+V) f_{n} \rightarrow H f$. (Actually we will show only weak convergence; but then suitable convex combinations of the $H f_{n}$ will converge strongly.)

We have the formula

$$
(-\Lambda+V) f_{n}=\phi(-\Lambda+V) g_{n}-g_{n} \Lambda \phi-2(a \nabla \phi) \cdot \nabla g_{n} .
$$

The first two terms on the right converge in norm to $\phi H f=H f$ and $-f \Lambda \phi=0$ respectively. We must show that the remaining term converges to 0 at least weakly. It obviously converges to 0 in the sense of distributions, so it is enough to show that the sequence of $L^{2}$ norms $\left\|(\alpha \nabla \phi) \cdot \nabla g_{n}\right\|$ is uniformly bounded. This amounts to showing that the sequence $\left\|\nabla g_{n}\right\|$ is bounded.

Now, because of the inequality $-\Delta \leqq$ const. $-\Lambda$, we have

$$
\begin{aligned}
\left\|\nabla g_{n}\right\|^{2} & =\left(-\Delta g_{n}, g_{n}\right) \leqq c\left(-\Lambda g_{n}, g_{n}\right) \\
& \leqq c\left((-\Lambda+U) g_{n}, g_{n}\right) .
\end{aligned}
$$

The right side of (2) converges to $c\left(H_{1} f, f\right)$. Hence the sequence $\left\|\nabla g_{n}\right\|$ is bounded.

We now consider the wave equation

$$
\frac{d^{2} u}{d t^{2}}=(\Lambda-V-\beta(t)) u(t) \text {. }
$$

Our aim is to show that under suitable hypotheses on $V$, equation (3) has global solutions. We first turn to the case of compact manifolds $M$, where existence of solutions is immediate from our earlier work on essential self-adjointness of $-\Lambda+V$. Here our intent is to establish support properties of solutions to (3). We treat the timeindependent case first.

Proposition 4.3. Let $M$ be compact Riemannian manifold. Let $\Lambda$ and $V$ be as in Proposition 4.1. Write $H$ for $\overline{-\Lambda+V}$. Consider the differential equation

$$
d^{2} u / d t^{2}=-H u(t), \quad u(0), \dot{u}(0) \in C^{\infty}(M) .
$$


By the results of $\S 3$ we know that (4) has a global solution $u(t)$.

Claim: This solution has the same support properties as the "free" equation $(V=0)$. That is, if the initial data are supported in the ball $B_{r}$, then, for all $t \geqq 0, u(t)$ is supported in the ball $B_{t_{*} r}$.

Proof. Basically this follows from the Trotter product formula as in the analogous case of Dirac operators in $\S 2$. First, suppose that $V$ is bounded. Without loss of generality we may assume $-\Lambda \geqq 1$. Consider, on the space $\boldsymbol{H}=\mathscr{D}(\sqrt{-\Lambda}) \oplus \mathscr{H}$, the operators

$$
A=\left[\begin{array}{ll}
0 & I \\
\bar{\Lambda} & 0
\end{array}\right] \quad B=\left[\begin{array}{cc}
0 & 0 \\
-V & 0
\end{array}\right] \text {. }
$$

Note that $B$ is a bounded operator on $\boldsymbol{H}$. If $\psi(t)=\left[\begin{array}{c}u(t) \\ \dot{u}(t)\end{array}\right]$ where $u$ is the unique solution to (4) we have $\psi(t)=e^{t(A+B)} \psi(0)$. Moreover, by the Trotter product formula,

$$
\psi(t)=\lim _{n \rightarrow \infty}\left(e^{t / n A} e^{t / n^{B}}\right)^{n} \psi(0) .
$$

Now $e^{t / n B}$ does not expand support, while if $\phi$ is supported in $B_{r}$, $e^{t / n A} \phi$ is supported in $B_{t / n * R}$. Hence the right side of (5) is supported in $B_{t / n_{*} \cdots * t / n * r}=B_{t * r}$. The same is true of the limit $\psi(t)$.

Now consider the general case: $V=U-W, v, W \geqq 0, U \in L^{2}(M)$, $W \in L^{p}(M)$. Then $H=\overline{-\Lambda+V}$ is semibounded, and the solution to (4) is given by

$$
u(t)=\cos (t \sqrt{H}) u(0)+\frac{\sin (t \sqrt{H})}{\sqrt{H}} \dot{u}(0) .
$$

Consider the cut-off potentials: $V_{n}(x)=V(x)$ if $|V(x)| \leqq n, V_{n}(x)=0$ otherwise. Let $H_{n}=-\overline{\Lambda+V_{n}}$. The operators $H_{n}$ are uniformly bounded below, and $H_{n} \rightarrow H$ pointwise on $\mathscr{D}(\Lambda) \cap \mathscr{D}(V)$, a core for $H$. Hence by [10, Th. VIII. 20] it follows that $\cos t \sqrt{\bar{H}_{n}} \rightarrow \cos t \sqrt{H}$ and

$$
\sin \left(t \sqrt{\bar{H}_{n}}\right) / \sqrt{\bar{H}_{n}} \longrightarrow \sin (t \sqrt{\bar{H}}) / \sqrt{\bar{H}}
$$

in the strong operator topology. That is, if $u_{n}(t)$ is the solution to (4) with $H$ replaced by $H_{n}$, then $u_{n}(t) \rightarrow u(t)$ for all $t$. By the first part of the proof we know that $u_{n}(t)$ is supported in $B_{t * r}$. Hence $u(t)$ is supported in $B_{t_{*} r}$.

Next we establish the same result for the time-dependent case.

Proposition 4.4. Let $M$ be a compact Riemannian manifold. Let $\Lambda$ and $V$ be as in Proposition 4.1. Let $\beta(t)$ be a differentiable 
real function of $t$. Write $H=-\overline{\Lambda+V}$. Then the equation

$$
d^{2} u / d t^{2}=-H u(t)-\beta(t) u(t), \quad u(0), \dot{u}(0) \in C^{\infty}(M)
$$

has a unique global solution $u(t)$. This solution satisfies the energy equation

$$
d / d t\left\{\|\dot{u}(t)\|^{2}+((H+\beta(t)) u(t), u(t))\right\}=\beta^{\prime}(t)\|u(t)\|^{2} .
$$

Moreover if the initial data are supported in the ball $B_{r}$, then, for $t \geqq 0, u(t)$ is supported in $B_{t_{*}}$.

Proof. Existence and uniqueness of the solution $u(t)$, as well as the energy equation (7), all follow from the results of $\S 3$. We must establish the support properties of $u(t)$.

Without loss of generality we may assume $H \geqq 1$. Then consider, on the space $\boldsymbol{H}=\mathscr{D}\left(H^{1 / 2}\right) \oplus \mathscr{H}$, the operators

$$
A=\left[\begin{array}{cc}
0 & I \\
-H & 0
\end{array}\right] \text { and } B(t)=\left[\begin{array}{cc}
0 & 0 \\
-\beta(t) & 0
\end{array}\right]
$$

If $\psi(t)=\left[\begin{array}{l}u(t) \\ \dot{u}(t)\end{array}\right]$ we know that $\psi$ satisfies the integral equation

$$
\psi(t)=e^{t A} \psi(0)+\int_{0}^{t} e^{(t-\tau) A} B(\tau) \psi(\tau) d \tau
$$

Moreover, the series expansion from iterating (8) converges because $\|B(\tau)\|$ is bounded on any bounded interval, so that we have the formula

$$
\psi(t)=e^{t A} \psi(0)+\sum_{n=1}^{\infty} \int_{\Delta_{m}} e^{\left(t-\tau_{1}\right) A} B\left(\tau_{1}\right) e^{\left(\tau_{1}-\tau_{2}\right) A} \cdots e^{\tau_{n} A} \psi(0) d \tau_{1} \cdots d \tau_{n}
$$

where $\Delta_{n}$ is the region $0 \leqq \tau_{n} \leqq \tau_{n-1} \leqq \cdots \leqq \tau_{1} \leqq t$. It is straightforward to check that each term in this series is supported in $B_{t * r}$ if $\psi(0)$ is supported in $B_{r}$. This follows from the fact that the operators $B(\tau)$ do not expand support, while, by Proposition 4.3 , the operators $e^{(t-\tau) A}$ expand support from $B_{r}$ to $B_{(t-\tau)_{*} r}$.

We can now extend the above results to the case of a complete (not necessarily compact) Riemannian manifold $M$.

THEOREM 4.5. Let $M$ be a complete Riemannian manifold, dimension d. Let $\Lambda$ be a second-order elliptic differential operator on $M$ of the form $\Lambda u=\nabla \cdot(a \nabla u)$. Define the propagation speed $c(r)$ associated with $M$ as indicated above, and assume that the integral $\int^{\infty} d r / c(r)$ diverges. 
Let $V$ be a real function on $M$ of the form $V=U-W$ where $U, W \geqq 0, U \in L_{1 \mathrm{loc}}^{2}(M), V \in L_{\mathrm{loc}}^{p}(M)$, and $p$ is $M$-canonical.

Let $H=\overline{-\Lambda+V}$.

Let $\beta(t)$ be a real differentiable function of $t$.

Then the differential equation

$$
d^{2} u / d t^{2}=-H u(t)-\beta(t) u(t), \quad u(0), \dot{u}(0) \in C_{0}^{\infty}(M)
$$

has a global solution $u(t)$. This solution satisfies the energy equation (7) above. Moreover, $u(t)$ is compactly supported for all $t$; if the initial data are supported in the ball $B_{r}$, then $u(t)$ is supported in $B_{t * r}$.

Finally, let $H_{0}$ denote the closure of the restriction of $H$ to $C_{0}^{\infty}(M)$. Then, for all $t$, the solution $u(t)$ belongs to $\mathscr{D}\left(H_{0}\right)$ (and of course $\left.H_{0} u(t)=H u(t)\right)$.

Proof. The idea of the proof is to use the preceding theorems to construct local solutions of (10) on an increasing sequence of compact subsets of $M$. The finite propagation speed guarantees that these fit together to define a global solution. The details follow.

Suppose that $u(0), \dot{u}(0)$ are $C^{\infty}$ functions supported in the ball $B_{r}$. For each positive integer $n$ let $\Omega_{n}$ be a compact subset of $M$ with smooth boundary which contains $\bar{B}_{n * r}$ in its interior. Finally, let $M_{n}$ be a compact Riemannian manifold (without boundary) in which $\Omega_{n}$ is isometrically embedded. (For example, let $M_{n}$ be the double covering of a neighborhood of $\Omega_{n}$; it is easy to see via a partition of unity argument that the Riemannian metric on $\Omega_{n}$ extends to a Riemannian metric on $M_{n}$. The precise choice of $M_{n}$ is immaterial.)

We extend the operator $\Lambda$ on $\Omega_{n}$ to be elliptic on $M_{n}$; call the extension $\Lambda_{n}$. The functions $U, W$, and $V$ may be extended to $M_{n}$ by defining them to be 0 outside $\Omega_{n}$. Since $\Omega_{n}$ has compact closure, we have $U_{n} \in L^{2}\left(M_{n}\right), W_{n} \in L^{p}\left(M_{n}\right)$. Finally the functions $u(0), \dot{u}(0)$ are extended by setting them equal to 0 outside $\Omega_{n}$.

We define $H_{n}=\overline{-\Lambda}_{n}+V_{n}$. It follows from Proposition 4.4 that the wave equation (10), with $H_{n}$ replacing $H$, has a unique global solution $u_{n}(t)$ in $L^{2}\left(M_{n}\right)$, and the energy equation (7) is satisfied. Moreover, for $|t|<n, u_{n}(t)$ is supported in the ball $B_{n * r}$, so for this range of $t$ we identify $u_{n}(t)$ with a function in $L^{2}(M)$. It follows from Corollary 4.2 that, for $|t|<n, u_{n}(t) \in \mathscr{D}\left(H_{0}\right)$, and $H_{0} u_{n}(t)=$ $H_{n} u_{n}(t)$.

Now suppose $m<n$, and $|t|<m$. From the uniqueness of solutions to the wave equation on $M_{m}$ it is clear that $u_{m}(t) \equiv u_{n}(t),|t|<$ $m$. Hence the functions $\left\{u_{n}\right\}_{n=1}^{\infty}$ fit together to define a global function 
$u(t) \in L^{2}(M)$ which has all the asserted properties.

We now come to the main theorem.

THEOREM 4.6. Let $M$ be a complete Riemannian manifold. Let $\Lambda$ be a second-order elliptic differential operator as in Theorem 4.5. We assume the integral $\int_{0}^{\infty} d r / c(r)$ diverges. Then define $r: M \rightarrow \boldsymbol{R}^{+}$ by $r(x)=d(x, 0)$, where 0 is the fixed origin in $M$, and set $\theta(r)=$ $\theta(r(x))=\int_{0}^{r(x)} d s / c(s)$. Suppose that $V=U-W$ as in Theorem 4.5. Define $H_{1}=-\Lambda+V$ on the domain $C_{0}^{\infty}(M)$. Assume that for some constants $a, b$ we have the inequality

$$
H_{1}=-\Lambda+V \geqq-\left(a+b \theta(\boldsymbol{r})^{2}\right) .
$$

Then $H_{1}$ is essentially self-adjoint.

Proof. We shall employ a simplified version of an argument of Kato [9].

First note that by using a change of scale if necessary, we can assume that the constant $b$ in (11) equals 1 . Also, the constant $a$ can be absorbed into the potential $V$, so we may assume that $a=0$.

We have to show that, for some positive $\lambda$, the equations $H_{0}^{*} \psi=$ $\pm i \lambda \psi$ have no nonzero solutions. It turns out that $\lambda=18$ is a convenient value. So suppose $H_{0}^{*} \psi=-i \cdot 18 \psi$.

Given $f \in C_{0}^{\infty}(M)$, we know by Theorem 4.5 with $\beta(t)=4 t^{2}$ that there is a global solution $u(t)$ of the equation

$$
d^{2} u / d t^{2}=-\left(H_{0}+4 t^{2}\right) u(t), \quad u(0)=f, \quad \dot{u}(0)=0 .
$$

Moreover, this solution obeys the energy equation

$$
\frac{d}{d t}\left\{\|\dot{u}(t)\|^{2}+\left(\left(H_{0}+4 t^{2}\right) u(t), u(t)\right)\right\}=8 t\|u(t)\|^{2} .
$$

Hence we have, for $t>r$,

$$
\|\dot{u}(t)\|^{2}+\left(\left(H_{0}+4 t^{2}\right) u(t), u(t)\right)=C_{r}+\int_{r}^{t} 8 s\|u(s)\|^{2} d s
$$

where $C_{r}$ is the value of the left side when $t=r$. Now suppose that the initial value $f$ is supported in the ball $B_{R}$. Then we know that $u(t)$ is supported in the ball $B_{t * R}$ where $t * R=\theta^{-1}(t+\theta(R))$. Accordingly, by (11) (with $a=0, b=1$ ) we have

$$
\begin{aligned}
\left(\left(H_{0}+4 t^{2}\right) u(t), u(t)\right) & \geqq\left[4 t^{2}-\theta(t * R)^{2}\right]\|u(t)\|^{2} \\
& =\left[4 t^{2}-(t+\theta(R))^{2}\right]\|u(t)\|^{2} \\
& \geqq t^{2}\|u(t)\|^{2}
\end{aligned}
$$


provided that $t \geqq r=2 \theta(R)$.

Equations (14) and (15) give the inequality

$$
t^{2}\|u(t)\|^{2} \leqq C_{r}+\int_{r}^{t} 8 s\|u(s)\|^{2} d s, \quad t \geqq r=2 \theta(R)
$$

from which it follows, by Gronwall's method, that $\|u(t)\|=0\left(t^{3}\right)$. Now return to our $\psi$ with $H_{0}^{*} \psi=-18 i \psi$ and define

$$
F(t)=(\psi, u(t)) \text {. }
$$

Then $F(0)=(\psi, f), \dot{F}(0)=0$, and

$$
\begin{aligned}
\ddot{F}(t)=(\psi, \ddot{u}(t)) & =\left(\psi,-H_{0} u(t)\right)-4 t^{2}(\psi, u(t)) \\
& =-\left(H_{0}^{*} \psi, u(t)\right)-4 t^{2}(\psi, u(t)),
\end{aligned}
$$

or

$$
\ddot{F}(t)=\left(18 i-4 t^{2}\right) F(t) .
$$

Since $F$ is a solution of (16) with $\dot{F}(0)=0$, it follows that $F(t)=$ $k\left(t^{4}-(3 / 2) i t^{2}-(3 / 16)\right) e^{i t^{2}}$ for some constant $k$. But since $\|u(t)\|=$ $0\left(t^{3}\right)$ we must have $F(t)=0\left(t^{3}\right)$, and thus $k=0$. In particular $F(0)=$ $(\psi, f)=0$. Hence $\psi$ is orthogonal to $C_{0}^{\infty}(M)$ and $\psi=0$.

A similar argument works with $-18 i$ replaced by $18 i$. This concludes the proof.

\section{REFERENCES}

1. Yu. M. Berezanskii, Self-adjointness of elliptic operators with singular coefficients (in Russian), Ukrainian Math. J., 26 (1974), 579-590.

2. P. R. Chernoff, Essential self-adjointness of powers of generators of hyperbolic equations, J. Functional Analysis, 12 (1973), 401-414.

3. W. G. Faris, Essential self-adjointness of operators in ordered Hilbert space, Commun. Math. Phys., 30 (1973), 23-34.

4. - Self-Adjoint Operators, Lecture Notes in Mathematics No. 433, SpringerVerlag (1975).

5. K. Jörgens, Perturbations of the Dirac Operator, in "Conference on the Theory of Ordinary and Partial Differential Equations," Lecture Notes in Mathematics No. 380, Springer-Verlag (1972), 87-102.

6. H. Kalf, U.-W. Schmincke, J. Walter, and R. Wüst, On the Spectral Theory of Schrödinger and Dirac Operators with Strongly Singular Potentials, in "Spectral Theory and Differential Equations," Lecture Notes in Mathematics No. 448, Springer-Verlag (1975), 182-226.

7. T. Kato, Perturbation Theory for Linear Operators, Springer-Verlag, 1966.

8. T. Kato, Schrödinger operators with singular potentials, Israel J. Math., 13 (1972), 135-148.

9. T. Kato, A remark to the preceding paper by Chernoff, J. Functional Analysis, 12 (1973), 415-417.

10. M. Reed and B. Simon, Methods of Modern Mathematical Physics, I: Functional Analysis. II: Fourier Analysis, Self-Adjointness, Academic Press, 1973 and 1975. 
11. M. Schechter, Spectra of Partial Differential Operators, North Holland, 1971.

12. B. Simon, Essential self-adjointness of Schrödinger operators with positive potentials, Math. Ann., 201 (1973), 211-220.

Received February 2, 1977. Research partially supported by N. S. F. Grant MCS 74-19876. A02.

UNIVERSITY OF CALIFORNIA

BERKELEY, CA 94720 


\title{
PACIFIC JOURNAL OF MATHEMATICS
}

\section{EDITORS}

\author{
RICHARD ARENS (Managing Editor) \\ University of California \\ Los Angeles, CA 90024 \\ Charles W. Curtis \\ University of Oregon \\ Eugene, OR 97403 \\ C. C. MOORE \\ University of California \\ Berkeley, CA 94720
}

\section{J. DUGUNDJI}

Department of Mathematics

University of Southern California

Los Angeles, CA 90007

R. FINN and J. MILGRAM

Stanford University

Stanford, CA 94305

\section{ASSOCIATE EDITORS}
E. F. BECKENBACH
B. H. NeumanN
F. WOLF
K. YoSHIDA

\section{SUPPORTING INSTITUTIONS}

UNIVERSITY OF BRITISH COLUMBIA

CALIFORNIA INSTITUTE OF TECHNOLOGY

UNIVERSITY OF CALIFORNIA

MONTANA STATE UNIVERSITY

UNIVERSITY OF NEVADA, RENO

NEW MEXICO STATE UNIVERSITY

OREGON STATE UNIVERSITY

UNIVERSITY OF OREGON

OSAKA UNIVERSITY

\author{
UNIVERSITY OF SOUTHERN CALIFORNIA \\ STANFORD UNIVERSITY \\ UNIVERSITY OF HAWAII \\ UNIVERSITY OF TOKYO \\ UNIVERSITY OF UTAH \\ WASHINGTON STATE UNIVERSITY \\ UNIVERSITY OF WASHINGTON \\ $\stackrel{*}{*} \stackrel{*}{*} \stackrel{*}{*}$ AMERICAN MATHEMATICAL SOCIETY
}

The Supporting Institutions listed above contribute to the cost of publication of this Journal, but they are not owners or publishers and have no responsibility for its content or policies.

Mathematical papers intended for publication in the Pacific Jaurnal of Mathematics should be in typed form or offset-reproduced, (not dittoed), double spaced with large margins. Please do not use built up fractions in the text of your manuscript. You may however, use them in the displayed equations. Underline Greek letters in red, German in green, and script in blue. The first paragraph or two must be capable of being used separately as a synopsis of the entire paper. Items of the bibliography should not be cited there unless absolutely necessary, in which case they must be identified by author and Journal, rather than by item number. Manuscripts, in triplicate, may be sent to any one of the editors. Please classify according to the scheme of Math. Reviews, Index to Vol. 39. All other communications should be addressed to the managing editor, or Elaine Barth, University of California, Los Angeles, California, 90024.

The Pacific Journal of Mathematics expects the author's institution to pay page charges, and reserves the right to delay publication for nonpayment of charges in case of financial emergency.

100 reprints are provided free for each article, only if page charges have been substantially paid. Additional copies may be obtained at cost in multiples of 50 .

The Pacific Journal of Mathematics is issued monthly as of January 1966. Regular subscription rate: $\$ 7200$ a year (6 Vols., 12 issues). Special rate: $\$ 36.00$ a year to individual members of supporting institutions.

Subscriptions, orders for back numbers, and changes of address should be sent to Pacific Journal of Mathematics, 103 Highland Boulevard, Berkeley, California, 94708.

PUBLISHED BY PACIFIC JOURNAL OF MATHEMATICS, A NON-PROFIT CORPORATION

Printed at Kokusai Bunken Insatsusha (International Academic Printing Co., Ltd.). 8-8, 3-chome, Takadanobaba, Shinjuku-ku, Tokyo 160, Japan.

Copyright (C) 1975 by Pacific Journal of Mathematics Manufactured and first issued in Japan 


\section{Pacific Journal of Mathematics}

Vol. 72, No. 2

February, 1977

George E. Andrews, Plane partitions. II. The equivalence of the

Bender-Knuth and MacMahon conjectures ................. 283

Lee Wilson Badger, An Ehrenfeucht game for the multivariable quantifiers

of Malitz and some applications ......................... 293

Wayne C. Bell, A decomposition of additive set functions ............ 305

Bruce Blackadar, Infinite tensor products of $C^{*}$-algebras ............. 313

Arne Brøndsted, The inner aperture of a convex set .............. 335

N. Burgoyne, Finite groups with Chevalley-type components........... 341

Richard Dowell Byrd, Justin Thomas Lloyd and Roberto A. Mena, On the retractability of some one-relator groups .....................

Paul Robert Chernoff, Schrödinger and Dirac operators with singular potentials and hyperbolic equations .................... 361

John J. F. Fournier, Sharpness in Young's inequality for convolution ....... 383

Stanley Phillip Franklin and Barbara V. Smith Thomas, On the metrizability

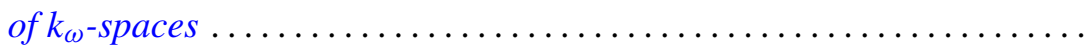

David Andrew Gay, Andrew McDaniel and William Yslas Vélez, Partially normal radical extensions of the rationals .................... 403

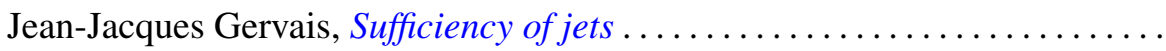

Kenneth R. Goodearl, Completions of regular rings. II . .

Sarah J. Gottlieb, Algebraic automorphisms of algebraic groups with stable maximal tori

Donald Gordon James, Invariant submodules of unimodular Hermitian forms.....

J. Kyle, $W_{\delta}(T)$ is convex.

Ernest A. Michael and Mary Ellen Rudin, A note on Eberlein compacts ...

Ernest A. Michael and Mary Ellen Rudin, Another note on Eberlein compacts ....

Thomas Bourque Muenzenberger and Raymond Earl Smithson, Fixed point theorems for acyclic and dendritic spaces.

Budh Singh Nashier and A. R. Rajwade, Determination of a unique solution of the quadratic partition for primes $p \equiv 1(\bmod 7)$.

Frederick J. Scott, New partial asymptotic stability results for nonlinear ordinary differential equations ....................

Frank Servedio, Affine open orbits, reductive isotropy groups, and dominant gradient morphisms; a theorem of Mikio Sato..........

D. Suryanarayana, On the distribution of some generalized square-full integers.................................. 